\section{Avaliação do rastreamento do câncer do colo do útero na Estratégia Saúde da Família no Município de Amparo, São Paulo, Brasil}

\author{
Assessment of the cervical cancer screening in \\ the Family Health Strategy in Amparo, \\ São Paulo State, Brazil
}

\author{
${ }^{1}$ Centro de Atenção \\ Integral à Saúde da Mulher \\ Universidade Estadual de \\ Campinas, Campinas, Brasil. \\ 2 Secretaria Municipal de \\ Saúde de Diadema, Diadema \\ Brasil. \\ 3 Faculdade de Ciências \\ Médicas, Universidade \\ Estadual de Campinas, \\ Campinas, Brasil. \\ Correspondência \\ L. C. Zeferino \\ Departamento de \\ Tocoginecologia, Faculdade \\ de Ciências Médicas, \\ Universidade Estadual de \\ Campinas. \\ Rua Vital Brasil 251, \\ Campinas, $S P$ \\ 13083-888, Brasil. \\ zeferino@hc.unicamp.br
}

\begin{abstract}
Uterine cervical cancer mortality has not been declining. The territorial distribution and registration offamilies in Brazil's Family Health Strategy help identify women that have performed a Pap smear or that have not had one for more than three years. This study analyzed whether cervical cancer screening in Amparo, São Paulo State, Brazil, made progress in complying with the prevailing guidelines during the seven years of experience with the Strategy. The annual examination rate remained high, with a slight trend towards greater intervals between follow-up tests. Distribution of tests tended to increase in the 40-59year age bracket and decrease in the over-60 age group, while annual coverage tended to decrease. The proportions of excess tests varied from $61.2 \%$ to $65.5 \%$. Concluding, the upgrading of cervical cancer screening was slight, and did not change the opportunistic pattern of follow-up tests. Considering that community health agents can act to increase the coverage of these measures, it is essential to train them for this work.
\end{abstract}

Mass Screening; Uterine Cervical Neoplasms; Family Health
Diama Bhadra Andrade Peixoto do Vale 1 Sirlei Siani Morais 1 Aparecida Linhares Pimenta 2 Luiz Carlos Zeferino 1,3

\section{Introdução}

O câncer do colo do útero é um importante problema de saúde pública, e sua incidência e mortalidade podem ser reduzidas por meio de programas de rastreamento efetivos 1 . Idealmente, o rastreamento do colo do útero deveria seguir um conjunto de ações programadas, com população e periodicidade definidas, o que tem sido denominado de programa organizado 2 . Todavia, é sabido que ainda predomina, em países como o Brasil, a realização de controles não relacionados com as normas estabelecidas, e sim com a procura ocasional dos serviços de saúde determinada por razões diversas que não o rastreamento do câncer do colo do útero. Essa modalidade tem sido designada de rastreamento oportunístico, e não tem sido eficiente em reduzir as taxas de incidência e mortalidade do câncer do colo do útero 2,3,4,5. O rastreamento oportunístico apresenta baixa cobertura, super-rastreia um pequeno grupo de mulheres e, portanto, é menos custo-efetivo 6,7 .

No Brasil, apesar de haver um programa de rastreamento do câncer do colo do útero, a taxa de mortalidade devido a esse câncer não tem reduzido ${ }^{8}$. As normas preconizadas para o rastreamento desse tipo de câncer no país seguem a tendência universal de não incluir prioritariamente as mulheres com menos de 25 anos e as com mais de 60, sendo que o intervalo ideal entre os controles é trienal ${ }^{9}$. 
Estratégias que busquem alterar esse cenário devem incluir a sistematização de um programa organizado, objetivando aumentar a cobertura das faixas etárias recomendadas para o rastreamento 2,3.

O Ministério da Saúde realizou em 2002-2003 um inquérito domiciliar que incluiu 15 capitais brasileiras e o Distrito Federal. Nesse estudo, a distribuição de mulheres de 25 a 59 anos que realizaram pelo menos um exame de Papanicolaou nos três anos anteriores à pesquisa variou de $32 \%$ no Rio de Janeiro a 63,4\% em Manaus, na população usuária do Sistema Único de Saúde (SUS) 10.

A Estratégia Saúde da Família (ESF) é um programa do sistema de saúde brasileiro que tem como objetivo reorientar o modelo assistencial. Ao incluir na sua prática a articulação entre a prevenção e a promoção da saúde, por meio da expansão e qualificação da atenção primária, gera um cenário favorável à reorganização do modo de rastreamento do câncer do colo do útero 11 .

Em comunidades com a ESF introduzida, espera-se que os agentes comunitários de saúde (ACS), por intermédio da adscrição da clientela, estabeleçam vínculo entre a equipe de referência e as famílias, objetivando uma maior resolubilidade da atenção. No contexto do rastreamento isso possibilitaria a identificação e busca ativa das pacientes sob risco e sem controles 11 .

Portanto, este estudo verificou se o rastreamento realizado no âmbito da ESF segue as recomendações do Ministério da Saúde. Os parâmetros analisados foram a periodicidade de realização dos exames, os grupos etários rastreados e a cobertura populacional do programa de rastreamento do câncer do colo do útero no Município de Amparo, Estado de São Paulo, que adota a ESF desde 1998.

\section{Métodos}

Este estudo foi realizado com mulheres usuárias do SUS de Amparo. Os sujeitos foram todas as mulheres que se submeteram ao exame citológico para rastreamento do câncer do colo do útero na ESF, de janeiro de 2001 a dezembro de 2007.

Amparo é um município do interior do Estado de São Paulo, situado a $127 \mathrm{~km}$ da capital, com uma projeção populacional para $2008 \mathrm{de}$ 68.227 habitantes, sendo 34.269 mulheres 12 . É considerado uma referência na implantação da ESF, sendo inclusive cenário de formação de recursos humanos em saúde pública, por meio de convênios estabelecidos com a Universidade Estadual de Campinas (UNICAMP). Em 2000, a cobertura populacional da ESF era de $50 \%$, e em 2006, $90 \% 12,13$.
A coleta de material para o exame de Papanicolaou foi realizada por profissional de enfermagem ou médico nas unidades básicas de saúde, e todos os exames foram enviados para o Laboratório de Citopatologia do Centro de Atenção Integral à Saúde da Mulher (CAISM) da UNICAMP. Os dados sobre os exames foram obtidos com base no sistema de informação do laboratório, que registra as informações a partir do formulário de requisição e resultados do exame de Papanicolaou. Foram excluídos os exames realizados com menos de um ano e aqueles em que as mulheres referiram exame anterior alterado, para não incluir desta forma os exames realizados em ambulatórios secundários. Também foram excluídos os exames sem informação da periodicidade.

O formulário de requisição e resultados disponibiliza informações sobre tempo desde o exame anterior (em anos) e idade da paciente (em anos completos), com base nos quais foram feitas análises relativas à periodicidade de realização dos exames e aos grupos etários assistidos. Para os cálculos de cobertura foram utilizados os dados de projeção populacional da Fundação Sistema Estadual de Análise de Dados (SEADE) 12.

O cálculo de excesso de exames de Papanicolaou foi realizado adotando-se como referencial o estudo de van Ballegooijen et al. 14, ajustado o cálculo aos dados disponíveis no sistema de informações do Laboratório de Citopatologia da UNICAMP. Tendo como referencial as normas do Ministério da Saúde 9, foi considerado excesso de exames:

- Todos os exames realizados fora do grupo etário alvo, ou seja, aqueles feitos em mulheres com até 24 anos ou com 60 anos ou mais.

- Intervalo desde o último controle menor do que três anos, ou seja, intervalos de um e dois anos.

Para estimar o total de exames em excesso, foi construída a seguinte fórmula $14:\{2 / 3(b-a)+1 / 2$ $\mathrm{c}+\mathrm{d}$ \}, sendo: $\mathrm{a}$ - primeiro controle de mulheres entre 25 e 59 anos, b - controle com intervalo de um ano de mulheres entre 25 e 59 anos, c controle com intervalo de dois anos de mulheres entre 25 e 59 anos, e d - todos os controles de mulheres com menos de 25 ou maior que 59 anos 9 .

Como o segundo controle da mulher deveria ser feito com intervalo de um ano, para corrigir o cálculo de excesso, dever-se-ia subtrair do total de exames com intervalo anual os exames realizados pela primeira vez (b - a). Tomando-se a periodicidade trienal como referência, em três anos as mulheres deveriam ter feito um controle. Para as mulheres que realizaram controles com um ano de intervalo, considerou-se dois terços como excesso após subtrair os casos de segundo controle $[2 / 3(b-a)]$, pois em três anos seriam 
realizados três controles, sendo que destes, somente um seria necessário. Para as mulheres que realizaram controle com dois anos de intervalo (c), considerou-se $50 \%$ como excesso, pois em três anos seriam feitos dois controles, sendo necessário somente um (1/2 c). Por fim, somou-se os exames realizados fora do grupo etário alvo (d). Os exames com três anos ou mais de intervalo não foram considerados como excesso.

Para análise estatística foi utilizado o teste de qui-quadrado de Cochran-Armitage para tendência.

\section{Resultados}

As freqüências de exames com intervalo de dois anos tenderam a aumentar $(\mathrm{p}<0,0001)$ entre 2001 e 2007, sendo o valor mais baixo $16,1 \%$ (2003) e o mais alto $24,1 \%$ (2007). Também tenderam a aumentar os exames com intervalo de 3 e 4-5 anos, sendo que a maior freqüência foi observada em 2007 ( $p<0,01)$. As freqüências dos exames realizados com intervalo maior que cinco anos e dos exames realizados pela primeira vez tenderam a diminuir, com $\mathrm{p}<0,0001$ para ambas as situações. Não houve variação significativa da freqüência dos exames realizados com intervalo de um ano no período analisado (Tabela 1).

As freqüências dos exames realizados no grupo etário de 40 a 59 anos tenderam a aumentar ( $p<0,0001$ ) entre 2001 e 2007, variando de 33 a $36,3 \%$. No grupo etário maior que 60 anos, as freqüências dos exames tenderam a diminuir ( $p<0,0001$ ) sendo os valores mais altos $11,7 \%$ em 2002 e 9,4\% em 2007. Não foram observadas diferenças significativas nas freqüências de exa- mes nos grupos etários de 0 a 24 anos e de 25 a 39 (p > 0,05) (Tabela 2)

Os percentuais de exames realizados por grupos etários tenderam à diminuição em todos os grupos etários analisados ( $p<0,0001)$, sendo os valores máximos observados no ano de 2002 $(9,9 \%, 27 \%, 28,3 \%$ e $17,1 \%$ para os grupos de 0 a 24, 25 a 39, 40 a 59 e maior que 60 anos, respectivamente) e os valores mínimos de 7,3\%, 18,5\%, $19,5 \%$ e $9,2 \%$, respectivamente, para os mesmos grupos (Tabela 3).

Os percentuais de exames em excesso no período analisado variaram entre $61,2 \%$, em 2002 , e $65,5 \%$, em 2005 , o que correspondeu a quase dois terços do total dos exames realizados ( $\mathrm{p}=0,068$ ) (Tabela 4).

\section{Discussão}

O presente estudo revelou que no município avaliado, apesar da implantação da ESF, o rastreamento do câncer do colo do útero permanece ocorrendo de modo predominantemente oportunístico. Mais de 50\% das mulheres de Amparo que fazem o exame citológico, realizam-no com uma periodicidade anual e isto não modificou ao longo dos anos. Entretanto, observou-se um aumento na freqüência dos exames com intervalo de dois a três anos e diminuição da freqüência dos exames realizados pela primeira vez e com intervalo superior a cinco anos, o que representa uma tendência ao espaçamento dos controles, aproximando discretamente das normas estabelecidas pelo Ministério da Saúde.

A maior parte dos exames foi realizada no grupo etário mais jovem, até 39 anos, o que é

Tabela 1

Variação anual da freqüência de exames citológicos de acordo com intervalo desde o último controle.

\begin{tabular}{|c|c|c|c|c|c|c|c|c|c|c|c|c|c|}
\hline \multirow[t]{2}{*}{ Ano } & \multicolumn{2}{|c|}{ 1o exame } & \multicolumn{2}{|c|}{1 ano } & \multicolumn{2}{|c|}{2 anos } & \multicolumn{2}{|c|}{3 anos } & \multicolumn{2}{|c|}{ 4-5 anos } & \multicolumn{2}{|c|}{$>5$ anos } & \multirow[t]{2}{*}{ Total } \\
\hline & $\mathbf{n}$ & $\%$ & $\mathrm{n}$ & $\%$ & $\mathbf{n}$ & $\%$ & $\mathbf{n}$ & $\%$ & $\mathbf{n}$ & $\%$ & $\mathbf{n}$ & $\%$ & \\
\hline 2001 & 675 & 13,7 & 2.650 & 53,7 & 941 & 19,1 & 292 & 5,9 & 171 & 3,5 & 206 & 4,2 & 4.935 \\
\hline 2002 & 871 & 14,6 & 3.214 & 53,9 & 976 & 16,4 & 386 & 6,5 & 259 & 4,3 & 256 & 4,3 & 5.962 \\
\hline 2003 & 744 & 13,2 & 3.337 & 59,0 & 911 & 16,1 & 307 & 5,4 & 200 & 3,5 & 158 & 2,8 & 5.657 \\
\hline 2004 & 642 & 11,6 & 3.299 & 59,7 & 989 & 17,9 & 277 & 5,0 & 174 & 3,1 & 144 & 2,6 & 5.525 \\
\hline 2005 & 545 & 10,0 & 3.210 & 58,8 & 1.045 & 19,1 & 330 & 6,0 & 181 & 3,3 & 149 & 2,7 & 5.460 \\
\hline 2006 & 424 & 8,8 & 2.781 & 57,7 & 948 & 19,7 & 342 & 7,1 & 194 & 4,0 & 129 & 2,7 & 4.818 \\
\hline 2007 & 394 & 8,8 & 2.300 & 51,2 & 1.081 & 24,1 & 320 & 7,1 & 235 & 5,2 & 164 & 3,6 & 4.494 \\
\hline Valor de $p$ * & \multicolumn{2}{|c|}{$<0,0001$} & \multicolumn{2}{|c|}{0,568} & \multicolumn{2}{|c|}{$<0,0001$} & \multicolumn{2}{|c|}{0,0048} & \multicolumn{2}{|c|}{0,0082} & \multicolumn{2}{|c|}{$<0,0001$} & \\
\hline Estatística & \multicolumn{2}{|c|}{$-10,0$} & \multicolumn{2}{|c|}{0,57} & \multicolumn{2}{|c|}{8,33} & \multicolumn{2}{|c|}{2,82} & \multicolumn{2}{|c|}{2,64} & \multicolumn{2}{|c|}{$-3,98$} & \\
\hline
\end{tabular}

* Teste de qui-quadrado de Cochran-Armitage para tendência. 
Tabela 2

Freqüência de exames realizados por grupos etários.

\begin{tabular}{|c|c|c|c|c|c|c|c|c|c|}
\hline \multirow[t]{2}{*}{ Ano } & \multicolumn{2}{|c|}{$0-24$ anos } & \multicolumn{2}{|c|}{ 25-39 anos } & \multicolumn{2}{|c|}{ 40-59 anos } & \multicolumn{2}{|c|}{$>60$ anos } & \multirow[t]{2}{*}{ Total } \\
\hline & $\mathbf{n}$ & $\%$ & $\mathbf{n}$ & $\%$ & $\mathrm{n}$ & $\%$ & $\mathbf{n}$ & $\%$ & \\
\hline 2001 & 1.051 & 21,3 & 1.648 & 33,4 & 1.666 & 33,8 & 570 & 11,6 & 4.935 \\
\hline 2002 & 1.252 & 21,0 & 1.994 & 33,4 & 2.021 & 33,9 & 695 & 11,7 & 5.962 \\
\hline 2003 & 1.256 & 22,2 & 1.883 & 33,3 & 1.867 & 33,0 & 651 & 11,5 & 5.657 \\
\hline 2004 & 1.198 & 21,7 & 1.798 & 32,5 & 1.938 & 35,1 & 591 & 10,7 & 5.525 \\
\hline 2005 & 1.192 & 21,8 & 1.733 & 31,7 & 1.960 & 35,9 & 575 & 10,5 & 5.460 \\
\hline 2006 & 1.020 & 21,2 & 1.577 & 32,7 & 1.750 & 36,3 & 471 & 9,8 & 4.818 \\
\hline 2007 & 932 & 20,7 & 1.510 & 33,6 & 1.629 & 36,2 & 423 & 9,4 & 4.494 \\
\hline Valor de $p$ * & \multicolumn{2}{|c|}{0,6538} & \multicolumn{2}{|c|}{0,4133} & \multicolumn{2}{|c|}{$<0,0001$} & \multicolumn{2}{|c|}{$<0,0001$} & \\
\hline Estatística & \multicolumn{2}{|c|}{$-0,45$} & \multicolumn{2}{|c|}{$-0,82$} & \multicolumn{2}{|c|}{4,32} & \multicolumn{2}{|c|}{$-4,79$} & \\
\hline
\end{tabular}

* Teste de qui-quadrado de Cochran-Armitage para tendência.

Tabela 3

Cobertura (\%) por grupo etário tendo como base o total anual de exames citológicos.

\begin{tabular}{|c|c|c|c|c|c|c|c|c|c|}
\hline \multirow[t]{2}{*}{ Ano } & \multicolumn{2}{|c|}{$0-24$ anos } & \multicolumn{2}{|c|}{ 25-39 anos } & \multicolumn{2}{|c|}{ 40-59 anos } & \multicolumn{2}{|c|}{$>60$ anos } & \multirow[t]{2}{*}{ Total } \\
\hline & $\mathrm{n}$ & $\%$ & $n$ & $\%$ & $n$ & $\%$ & $n$ & $\%$ & \\
\hline 2001 & 1.051 & 8,3 & 1.648 & 22,7 & 1.666 & 24,2 & 570 & 14,4 & 4.935 \\
\hline 2002 & 1.252 & 9,9 & 1.994 & 27,0 & 2.021 & 28,3 & 695 & 17,1 & 5.962 \\
\hline 2003 & 1.256 & 9,9 & 1.883 & 25,0 & 1.867 & 25,2 & 651 & 15,7 & 5.657 \\
\hline 2004 & 1.198 & 9,4 & 1.798 & 23,5 & 1.938 & 25,3 & 591 & 13,9 & 5.525 \\
\hline 2005 & 1.192 & 9,3 & 1.733 & 22,2 & 1.960 & 24,7 & 575 & 13,3 & 5.460 \\
\hline 2006 & 1.020 & 8,0 & 1.577 & 19,7 & 1.750 & 21,5 & 471 & 10,6 & 4.818 \\
\hline 2007 & 932 & 7,3 & 1.510 & 18,5 & 1.629 & 19,5 & 423 & 9,2 & 4.494 \\
\hline Valor de $p^{*}$ & \multicolumn{2}{|c|}{$<0,0001$} & \multicolumn{2}{|c|}{$<0,0001$} & \multicolumn{2}{|c|}{$<0,0001$} & \multicolumn{2}{|c|}{$<0,0001$} & \\
\hline Estatística & \multicolumn{2}{|c|}{$-5,4$} & \multicolumn{2}{|c|}{$-12,15$} & \multicolumn{2}{|c|}{$-11,34$} & \multicolumn{2}{|c|}{$-11,43$} & \\
\hline
\end{tabular}

* Teste de qui-quadrado de Cochran-Armitage para tendência.

Fonte: Fundação Sistema Estadual de Análise de Dados (SEADE) 12.

compatível com o rastreamento oportunístico. Não houve modificação do percentual de exames realizados entre as mulheres com até 39 anos, porém, este percentual aumentou entre as de 40 a 59 anos e reduziu nas mulheres com 60 anos ou mais, sugerindo que há uma tendência em priorizar os grupos etários considerados de risco (25 a 59 anos) 9 .

A diminuição da freqüência dos exames realizados pela primeira vez não necessariamente retrata menor capacidade da ESF em recrutar as mulheres. Observou-se que um pouco mais de $20 \%$ das mulheres vinham fazendo os controles antes dos 20 anos. Tendo em vista que o Ministério da Saúde recomenda que se inicie os controles aos 25 anos, a correção dessa distorção implicaria reduzir temporariamente a inclusão de mulheres realizando exames pela primeira vez. Se essa suposição for verdadeira, nos próximos anos a freqüência de mulheres realizando controles pela primeira vez deverá aumentar.

A cobertura populacional com base nos exames realizados em cada ano tendeu a diminuir em todos os grupos etários analisados. Considerando que as normas do Ministério da Saúde preconizam periodicidade trienal, a cobertura que importa corresponde ao percentual de mulheres que fizeram pelo menos um exame nos últimos três anos. Assim, apesar da diminuição observada da cobertura anual, a cobertura trienal foi favorecida pelo aumento do percentual de mulheres que realizaram controles com intervalos de dois e três anos. 
Tabela 4

Exames em excesso por ano, de acordo com as normas do Ministério da Saúde *.

\begin{tabular}{|c|c|c|c|c|c|c|c|c|}
\hline Ano & $\begin{array}{c}\text { a: } \\
\text { primeiro } \\
\text { controle }\end{array}$ & $\begin{array}{c}\text { b: } \\
\text { intervalo } \\
\text { de até } 1 \text { ano }\end{array}$ & $\begin{array}{c}\text { c: } \\
\text { intervalo } \\
\text { de } 2 \text { anos }\end{array}$ & $\begin{array}{c}\mathrm{d}: \\
\text { mulheres }< \\
25 \text { anos; } \geq \\
60 \text { anos }\end{array}$ & $\begin{array}{l}\text { Exames em } \\
\text { excesso } \\
\begin{array}{c}{[2 / 3(b-a)+} \\
1 / 2 c+d] \\
n(\%)\end{array}\end{array}$ & (IC95\%) & $\begin{array}{l}\text { Exames de } \\
\text { acordo com } \\
\text { as normas * }\end{array}$ & $\begin{array}{l}\text { Total de } \\
\text { exames } \\
\text { realizados }\end{array}$ \\
\hline 2001 & 211 & 1.945 & 674 & 1.621 & $3.114(63,1)$ & $(61,8-64,4)$ & 1.821 & 4.935 \\
\hline 2002 & 301 & 2.315 & 715 & 1.947 & $3.647(61,2)$ & $(59,9-62,4)$ & 2.315 & 5.962 \\
\hline 2003 & 243 & 2.390 & 640 & 1.907 & $3.658(64,7)$ & $(63,4-65,9)$ & 1.999 & 5.657 \\
\hline 2004 & 215 & 2.384 & 698 & 1.789 & $3.584(64,9)$ & $(63,6-66,1)$ & 1.941 & 5.525 \\
\hline 2005 & 147 & 2.314 & 731 & 1.767 & $3.577(65,5)$ & $(64,3-66,8)$ & 1.883 & 5.460 \\
\hline 2006 & 94 & 2.067 & 692 & 1.491 & $3.152(65,4)$ & $(64,1-66,8)$ & 1.666 & 4.818 \\
\hline 2007 & 100 & 1.705 & 787 & 1.355 & $2.819(62,7)$ & $(61,3-64,1)$ & 1.675 & 4.494 \\
\hline Valor de $p^{\star \star}$ & & & & & 0,0680 & & & \\
\hline Estatística & & & & & 1.825 & & & \\
\hline
\end{tabular}

* Normas do Ministério da Saúde: exames de 25-59 anos; controles trienais após dois resultados negativos com intervalo de um ano;

** Teste de qui-quadrado de Cochran-Armitage para tendência.

A cobertura populacional observada foi maior no grupo etário 25 a 39 anos, variando de 18,5\% a $27 \%$. Como mais de $50 \%$ dos controles foram com um ano de intervalo, a cobertura trienal está muito prejudicada pelo excesso de exames em mulheres consideradas protegidas de acordo com as normas do Ministério da Saúde. Não foi calculada a cobertura global do rastreamento do câncer do colo do útero na população de Amparo, pois não foram incluídas as mulheres que realizaram este controle nas clínicas privadas e nem aquelas que poderiam ter realizado o exame em outros municípios.

Dois municípios do Rio Grande do Sul 15,16 mostraram taxas de cobertura acima de $80 \%$ para intervalo de três anos, o que pode ser considerado um excelente resultado quando comparado com dados de países desenvolvidos. Para tal, foram realizadas pesquisas de base populacional, por meio de amostragem por conglomerados, que é a metodologia adequada para estimar a cobertura. A limitação desse método é que também são incluídas as mulheres assistidas pelas clínicas privadas que, provavelmente, tendem a elevar a taxa de cobertura.

A elevada freqüência de exames em excesso (maior que $60 \%$ ) é tipicamente observada em programas de rastreamento oportunístico 2,3,6,17. Ao longo dos sete anos foram realizados aproximadamente 36.851 exames citológicos, sendo que 23.551 não foram de acordo com as normas do Ministério da Saúde e portanto podem ser considerados como exames em excesso. To- mando a projeção da população feminina para o ano de 2007 que é de 33.802 mulheres, sendo 16.534 no grupo de 25 a 59 anos, se os exames fossem realizados de acordo com as normas do Ministério da Saúde todas as mulheres do grupo etário recomendado teriam realizado exames 2,2 vezes em sete anos, ou seja, seria atingida $100 \%$ de cobertura trienal no período. Desse modo o rastreamento poderia se tornar mais efetivo, sem a necessidade de expandir a capacidade assistencial.

Reduzir a freqüência dos exames em excesso significa otimizar os recursos disponíveis. $\mathrm{O}$ objetivo não deve ser a redução do número de coletas, e sim desenvolver estratégias para que aquelas mulheres sob maior risco 9,18,19 de desenvolver câncer do colo do útero tenham acesso ao sistema, seja por demanda espontânea ou por busca ativa.

A incidência e mortalidade pelo câncer do colo do útero podem ser quase que totalmente prevenidas por ações de saúde pública, desde que o planejamento destas ações esteja em consonância com o conhecimento científico atual, e a execução das mesmas seja corretamente gerenciada 1,2,3,20. A análise do modo de rastreamento e do impacto dessas ações pode gerar um conjunto de indicadores fundamentais para se obter maior eficiência deste programa em termos de saúde pública ${ }^{21}$.

É conhecimento consolidado que o principal fator que limita a detecção do câncer do colo do útero é a baixa cobertura, em geral devida à falta 
de regularidade na coleta do exame de Papanicolaou 19,22,23. Outro fator que favorece a ineficiência de um programa não organizado é a dificuldade na confirmação diagnóstica, seguimento e tratamento dos casos rastreados 19,24.

Infelizmente, os sistemas de informação do SUS são baseados em procedimentos e não na pessoa, como é o caso do SISCOLO (Sistema de Informação do Câncer do Colo do Útero), que registra os exames citológicos com diagnósticos alterados realizados no Brasil, controlando diversas variáveis, mas que não permite registrar a história do rastreamento dessas mulheres e, conseqüentemente, não permite identificar quem está sem controle ou o fez há três anos ou mais.

Além da dificuldade em se obter um histórico do rastreamento individual das mulheres que permitiria propor estratégias de seguimento, outro obstáculo é a dificuldade de acesso que algumas mulheres têm aos serviços de saúde 18 .

A busca por um sistema de informação longitudinal baseado na pessoa, como propõe o cartão SUS, poderia identificar as mulheres expostas a risco que estariam há mais de três anos sem fazer exame citológico. A estratégia de recrutamento sistemático, ou busca ativa, não apenas melhora a cobertura dos programas, mas também tende a reduzir as iniqüidades no rastreamento de populações desfavorecidas $25,26,27,28$.

A terrritorialização e o cadastramento das famílias, práticas conceituais da ESF, favorecem que os ACS identifiquem a população de mulheres-alvo e convoquem aquelas que nunca fizeram o exame ou que estão há mais de três anos sem fazê-lo. Todavia, os resultados deste estudo mostraram que essa ação ainda é deficiente, uma vez que ao longo dos sete anos analisados de ESF observou-se algum ajuste no rastreamento do câncer do colo do útero, sem no entanto modificar a modalidade oportunística para um programa organizado. Portanto, essa ação totalmente compatível com o papel dos ACS deve ser introduzida ou reforçada nos programas de capacitação destes profissionais e, obviamente, deve ser uma preocupação de toda a equipe da ESF.

\section{Conclusão}

O rastreamento realizado no âmbito da ESF no município analisado não segue as recomendações do Ministério da Saúde. É necessário destacar no cadastramento das famílias por meio da ESF as mulheres-alvo do rastreamento do câncer do colo do útero e registrar os controles realizados, evitando a concentração excessiva de exames nas mulheres jovens e naquelas que mais freqüentam os serviços de saúde, favorecendo as que precisariam de uma ação ativa dos ACS na identificação, convencimento e realização dos exames periódicos, minimamente atendendo às normas do Ministério da Saúde.

\section{Resumo}

A mortalidade por câncer do colo uterino não está reduzindo. A terrritorialização e o cadastramento das famílias pela Estratégia Saúde da Família (ESF) favorece que se identifique as mulheres que nunca fizeram o exame citológico ou que estão há mais de três anos sem fazê-lo. Este estudo analisou se o rastreamento do câncer do colo uterino em Amparo, Estado de São Paulo, Brasil, avançou no sentido de seguir as recomendações vigentes ao longo de sete anos da ESF. A periodicidade anual manteve-se alta, com discreta tendência ao espaçamento dos controles. A distribuição de exames tendeu a aumentar no grupo etário
40-59 anos e a diminuir no grupo etário com mais de 60 anos, e a cobertura anual tendeu a diminuir. Os percentuais de exames em excesso variaram de 61,2\% a 65,5\%. Concluindo, a qualificação do rastreamento do câncer do colo do útero foi discreta e não modificou o padrão oportunístico dos controles. Considerando que os agentes comunitários de saúde podem atuar no sentido de aumentar a cobertura dessas ações, é fundamental capacitá-los para tal.

Programas de Rastreamento; Neoplasias do Colo do Útero; Saúde da Família 


\section{Colaboradores}

D. B. A. P. Vale e L. C. Zeferino foram responsáveis pela concepção do projeto, análise e interpretação dos dados, redação e revisão crítica do conteúdo. S. S. Morais colaborou com o desenho do estudo, a análise estatística dos dados e revisão crítica do conteúdo do artigo. A. L. Pimenta colaborou na revisão crítica do conteúdo do artigo.

\section{Referências}

1. Quinn M, Babb P, Jones J, Allen E. Effect of screening on incidence of and mortality from cancer of cervix in England: evaluation based on routinely collected statistics. BMJ 1999; 318:904-8.

2. Madlensky L, Goel V, Polzer J, Ashbury FD. Assessing the evidence for organised cancer screening programmes. Eur J Cancer 2003; 39:1648-53.

3. Zeferino LC, Derchain SF. Cervical cancer in the developing world. Best Pract Res Clin Obstet Gynaecol 2006; 20:339-54.

4. Zeferino LC, Pinotti JA, Jorge JP, Westin MCA, Tambascia JK, Montemor EBL. Organization of cervical cancer screening in Campinas and surrounding region, São Paulo State, Brazil. Cad Saúde Pública 2006; 22:1909-14.

5. Zeferino LC. The challenge of reducing mortality due to cervical cancer. Rev Bras Ginecol Obstet 2008; 30:213-5.

6. Adab P, McGhee SM, Yanova J, Wong CM, Hedley AJ. Effectiveness and efficiency of opportunistic cervical cancer screening comparison with organized screening. Med Care 2004; 42:600-9.
7. Goldie SJ, Gaffikin L, Goldhaber-Fiebert JD, Gordillo-Tobar A, Levin C, Mahé C, et al. Cost-effectiveness of cervical-cancer screening in five developing countries. N Engl J Med 2005; 353:2158-68.

8. Instituto Nacional de Câncer. Atlas de mortalidade por câncer - série temporal 1990-2005. http:// mortalidade.inca.gov.br (acessado em Jan/2009).

9. Instituto Nacional de Câncer. Periodicidade de realização do exame preventivo do câncer do colo do útero: normas e recomendações do INCA. Rev Bras Cancerol 2002; 48:13-5.

10. Instituto Nacional de Câncer. Inquérito domiciliar sobre comportamentos de risco e morbidade referida de doenças e agravos não transmissíveis: Brasil, 15 capitais e Distrito Federal, 2002-2003. Rio de Janeiro: Instituto Nacional de Câncer; 2004.

11. Departamento de Atenção Básica, Ministério da Saúde. Atenção básica e a saúde da família. Brasília: Ministério da Saúde; 2004.

12. Fundação Sistema Estadual de Análise de Dados. Projeções populacionais. http://www.seade.gov. br/produtos/projpop/ (acessado em Jan/2009). 
13. Secretaria Municipal de Saúde, Prefeitura do Município de Amparo. Relatório de gestão da secretaria municipal de saúde de Amparo, 2006. Amparo: Secretaria Municipal de Saúde; 2007.

14. van Ballegooijen M, van den Akker-van Marle E, Patnick J, Lynge E, Arbyn M, Anttila A, et al. Overview of important cervical cancer screening process values in European Union (EU) countries, and tentative predictions of the corresponding effectiveness and cost-effectiveness. Eur J Cancer 2000; 36:2177-88.

15. Muller DK, Dias-da-Costa JS, Luz AMH, Olinto MTA. Cobertura do exame citopatológico do colo do útero na cidade de São Leopoldo, Rio Grande do Sul, Brasil. Cad Saúde Pública 2008; 24:2511-20.

16. Hackenhaar AA, Cesar JA, Domingues MR. Exame citopatológico de colo uterino em mulheres com idade entre 20 e 59 anos em Pelotas, RS: prevalência, foco e fatores associados à sua não realização. Rev Bras Epidemiol 2006; 9:103-11.

17. Freitas RA, Carvasan GA, Morais SS, Zeferino LC. Excessive Pap smears due to opportunistic cervical cancer screening. Eur J Gynaecol Oncol 2008; 29:479-82.

18. Brenna SM, Hardy E, Zeferino LC, Namura I. Conhecimento, atitude e prática do exame de Papanicolaou em mulheres com câncer de colo uterino. Cad Saúde Pública 2001; 17:909-14.

19. Priest P, Sadler L, Peters J, Crengle S, Bethwaite P, Medley G, et al. Pathways to diagnosis of cervical cancer: screening history, delay in follow up, and smears reading. BJOG 2007; 114:398-407.

20. Peto J, Gilham C, Fletcher O, Matthews FE. The cervical cancer epidemic that screening has prevented in the UK. Lancet 2004; 364:249-56.

21. Bottari CMS, Vasconcellos MM, Mendonça MHM. Câncer cérvico-uterino como condição marcadora: uma proposta de avaliação da atenção básica. Cad Saúde Pública 2008; 24 Suppl 1:S111-22.
22. Nygård JF, Nygård M, Skare GB, Thoresen SØ. Screening histories of women with CIN $2 / 3$ compared with women diagnosed with invasive cervical cancer: a retrospective analysis of the Norwegian Coordinated Cervical Cancer Screening Program. Cancer Causes Control 2005; 16:463-74.

23. Spayne J, Ackerman I, Milosevic M, Seidenfeld A, Covens A, Paszat L. Invasive cervical cancer: a failure of screening. Eur J Public Health 2008; 18:162-5.

24. Mubiayi N, Bogaert E, Boman F, Leblanc E, Vinatier D, Leroy JL, et al. Cytological history of 148 women presenting with invasive cancer. Gynecol Obstet Fertil 2002; 30:210-7.

25. Jibaja-Weiss ML, Volk RJ, Smith QW, Holcomb JD, Kingery P. Differential effects of messages for breast and cervical cancer screening. J Health Care Poor Underserved 2005; 16:42-52.

26. Morrell S, Taylor R, Zeckendorf S, Niciak A, Wain G, Ross J. How much does a reminder letter increase cervical screening among under-screened women in NSW? Aust N Z J Public Health 2005; 29:78-84.

27. Stein K, Lewendon G, Jenkins R, Davis C. Improving uptake of cervical cancer screening in women with prolonged history of non-attendance for screening: a randomized trial of enhanced invitation methods. J Med Screen 2005; 12:185-9.

28. Albuquerque KM, Frias PG, Andrade CLT, Aquino EML, Menezes G, Szwarcwald CL. Cobertura do teste de Papanicolaou e fatores associados a nãorealização: um olhar sobre o Programa de Prevenção do Câncer do Colo do Útero em Pernambuco, Brasil. Cad Saúde Pública 2009; 25 Suppl 2:S301-9.

Recebido em 29/Ago/2009

Versão final reapresentada em 22/Nov/2009

Aprovado em 14/Nov/2009 